\title{
A SEARCH FOR ASTROPHYSICAL POINT SOURCES OF 100 TEV GAMMA RAYS BY THE UMC COLLABORATION
}

\author{
T.A.McKay, ${ }^{b}$ A.Borione, ${ }^{b}$ M.Catanese, ${ }^{a}$ C.E.Covault,${ }^{b}$ J.W.Cronin, ${ }^{b}$ \\ B.E.Fick, ${ }^{b}$ K.G.Gibbs, ${ }^{b}$ K.D.Green,${ }^{a}$ S.Hauptfeld ${ }^{b}$ D.Kieda,${ }^{c}$ H.A. Krimm, ${ }^{b *}$ \\ N.M.Mascarenhas, ${ }^{b \dagger}$ J.Matthews, ${ }^{a}$ D.Müller, ${ }^{b}$ B.J.Newport,${ }^{b}$ D.Nitz, ${ }^{a}$ \\ R.A.Ong, ${ }^{b}$ L.J.Rosenberg, ${ }^{b}$ D.Sinclair, ${ }^{a}$ J.C. Van Der Velde ${ }^{a}$ \\ ${ }^{a}$ Department of Physics, U. of Michigan, Ann Arbor, MI 48109 \\ ${ }^{b}$ Enrico Fermi Institute, U. of Chicago, Chicago, IL 60637 \\ ${ }^{c}$ Department of Physics, U. of Utah, Salt Lake City, UT 84112
}

\begin{abstract}
The CASA-MIA experiment is a very large extensive air shower detector with good angular resolution. This instrument has been used to search the entire visible sky for astrophysical point sources of $100 \mathrm{TeV}$ gamma rays. Approximately $90 \%$ of the isotropic charged cosmic ray background is rejected by measuring the muon content of the showers. Stringent limits are placed on the possible flux of $100 \mathrm{TeV}$ sources across a large part of the Northern sky.
\end{abstract}

\section{INTRODUCTION}

Gamma ray astronomy at energies in excess of $50 \mathrm{GeV}$ is limited to ground based experiments by the very low source fluxes involved. Because of the intervening atmosphere, ground based detection of gamma rays must be achieved indirectly, through detection of the extensive air showers which they create. This indirect detection has mixed consequences. Air showers have the attractive property of dispersing the effect of the primary particle. This allows air shower arrays to be fully efficient while instrumenting only about $1 \%$ of the total area. Without this property construction of a detector with $>10^{5} \mathrm{~m}^{2}$ detection area would be impossible. Unfortunately the air showers initiated by the isotropic charged cosmic ray background are difficult to distinguish from those initiated by gamma rays. Rejecting this background is the principal

\footnotetext{
"Center for Space Research, M.I.T., Cambridge, MA 02139

tNorman Bridge Laboratory, Cal.Tech. Pasadena, CA 91125
}

experimental challenge of ground based gamma ray astronomy.

Gamma rays from astrophysical point sources have now been confidently detected at energies up to at least $15 \mathrm{TeV}^{1,2}$ by experiments which measure the Cherenkov light emitted by air showers and reject the cosmic ray background with the imaging technique ${ }^{3}$. At higher energies (> 50-150 TeV, depending on altitude) the showers generated are sufficiently penetrating to reach the surface, where they may be detected directly.

\section{THE CASA-MIA DETECTOR}

The CASA-MIA detector is a two component extensive air shower array. The Chicago Air Shower Array (CASA) is a 1089 element surface array which detects the arrival direction, surface size, and core location of air showers. Each detector station contains four scintillation counters totalling $1.5 \mathrm{~m}^{2}$ and extensive local detection and data acquisition electronics. The stations are placed on a 15 
$\mathrm{m}$ square grid and enclose a total of $2.3 \times 10^{5}$ $\mathrm{m}^{2}$. The Michigan Anti (MIA) is an underground muon array consisting of $10242.5 \mathrm{~m}^{2}$ scintillation counters grouped in 16 'patches' of 64 counters each and buried beneath $3 \mathrm{~m}$ of earth. This earth is sufficient to stop the electromagnetic component of showers, so that MIA measures only the muon content of showers. CASA-MIA has been described in detail elsewhere $^{4,5}$.

The combined array is located on the U.S. Army Dugway Proving Ground (40.2 ${ }^{\circ} \mathrm{N}$, $112.8^{\circ} \mathrm{W}$ ) at an average atmospheric depth of $870 \mathrm{gcm}^{-2}$. It detects $100 \mathrm{TeV}$ air showers at a rate of more than $20 \mathrm{~Hz}$. More than half the array, 529 CASA stations and 8 MIA patches, were operated during array construction to obtain the data analyzed here.

The energy response of the array is determined by comparison of the measured cosmic ray flux to the previously measured fluxes. Corrections are made to account for the mixed primary particle composition and for differences between gamma ray and cosmic ray shower development. The array is $75 \%$ efficient for vertical gamma ray showers at an energy of about $60 \mathrm{TeV}$, and the median gamma ray shower energy for sources which pass overhead is $140 \mathrm{TeV}$.

\section{BACKGROUND REJECTION}

In searching for point sources of gamma rays the most important background rejection tool is angular resolution. Since the background is isotropic background rejection increases linearly as resolution improves. The angular resolution of CASA has been measured by observing the shadowing of the charged cosmic ray background by the Sun and Moon. The resolution is defined by the half angle of a bin which contains $63 \%$ of the signal from a point source $\left(\sigma_{63}\right)$. The Sun and Moon shadows indicate that $\sigma_{63}$ is $1.2^{\circ}$ for all data, improving to $0.5^{\circ}$ with increasing shower size.

Further rejection of background must rely on the intrinsic properties of the showers. Charged cosmic ray showers contain a hadronic core which produces many muons through pion decay. Gamma ray showers have no hadronic core, and consequently contain about 40 times fewer muons than similar cosmic ray showers. So background may be further rejected by selecting only 'muon poor' showers. The difficulty in implementing this technique is that there are only $10 \%$ as many muons as electrons in a shower, and the muon lateral distribution is quite wide. Even with MIA's $2500 \mathrm{~m}^{2}$ active area the average number of muons detected is only eight.

We determine the expected number of muons detected as a function of shower size, zenith angle, and core location, by parametrizing the average muon content of observed background showers. and compare this number to the observed number of muons. The definition of muon poor showers is chosen to optimize the signal sensitivity. If we observe $\leq 25 \%$ of the expected number of muons, the shower is muon poor. This selection rejects approximately $90 \%$ of the background. This rejection is limited by the fraction of showers in which the number of detected muons fluctuates to zero. Monte Carlo studies show that $70 \%$ of the gamma ray events are retained by this cut. Gamma rays are lost primarily because of accidental muon hits.

\section{ALL SKY SURVEY TECHNIQUE}

To search for gamma ray point sources we produce a map of the sky and search for localized excesses of showers. The map is generated by tiling the sky with bin centers spaced by $0.8^{\circ}$ in declination and $0.8^{\circ} / \cos$ (declination) in right ascension. We use bins spaced more closely than the resolution suggests to avoid a 
loss of sensitivity near bin edges. We construct two sky maps, one containing all events, and a second containing only muon poor events. Any real gamma ray source should appear with greater significance in the muon poor sky.

We generate the expected background map in the following manner. We measure the azimuth yield of the array. For each signal event, five background events are generated. Signal and background events increment all bins whose centers fall within $1.2^{\circ}$. Each background event has the same altitude as the signal event, but an azimuth selected randomly from the measured azimuth distribution. The five times oversampling of background means that fluctuations are dominated by signal variations. When the background is generated in this way a small $(0.5 \%)$ declination dependent systematic difference between signal and background remains. We confirm that this systematic is not due to a few point sources by observing that it is the same for all right ascensions and correct for it.

We search a region of the sky ranging from $8^{\circ}$ to $68^{\circ}$ in declination. This region contains 25126 individual signal bins. Since the sky is oversampled we expect the excess from a real source to be spread over four to seven bins. A real source should have a spatial distribution in keeping with the measured instrumental resolution. We search for potential sources by looking for groups of four or more bins, each with significance greater than $2.8 \sigma$. We have inserted artificial sources into the real sky background and found that this technique finds $\geq 4.2 \sigma$ sources with $90 \%$ efficiency.

Picking sources out of the full sky is difficult because of the large number of potential source bins. If a specific location is selected by independent means, flux sensitivity is typically a factor of 0.4 better.

\section{ALL SKY SURVEY RESULTS}

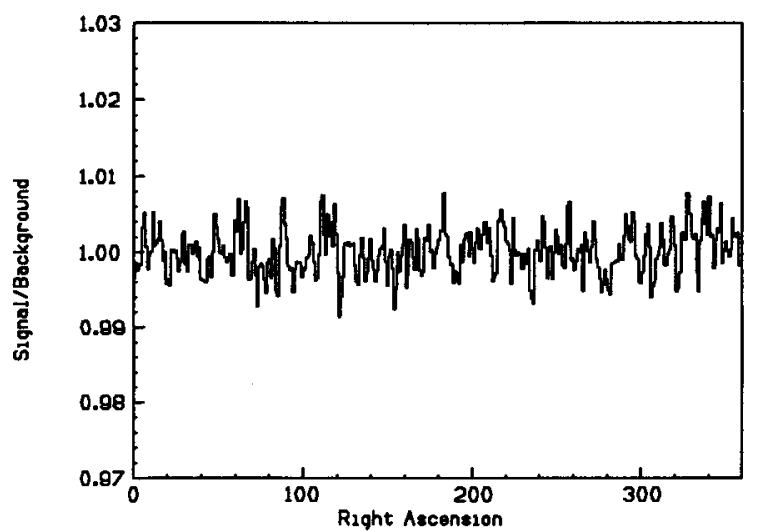

Figure 1. Ratio of signal to background is shown for a right ascension slice at declination $37.6^{\circ}$.

The data used in this search are drawn from more than 400 million array triggers recorded between 4 March 1990 and 22 April 1991 , a period of 414 days. 345 million of these events were large enough to be reconstructed. Source bins which pass overhead contain about 115,000 events, of which perhaps 12,000 are muon poor. The ratio of signal to background for a right ascension slice which passes overhead is shown in Figure 1.

We compare signal to background in each of the sky maps using the statistic of $\mathrm{Li}$ and $\mathrm{Ma}^{6}$. The distribution obtained for the muon poor sky is shown in Figure 2. Overlaid on the distribution is the best fit Gaussian, which has mean $-0.00048+/-0.00629$ and width 0.9925 $+/-0.0045$. A search of this sky finds no regions which pass the search cuts defined above in either sky map. Studies of a random sky lead us to expect 1.25 in each map from random fluctuations.

Since energetic astrophysical phenomena are so often transient, and since some previous reports of $100 \mathrm{TeV}$ gamma ray emission have been transient ${ }^{7}$, we also search for emission on short time scales. We accomplish this by searching the sky as described above on a daily basis. Sources pass overhead for only a 


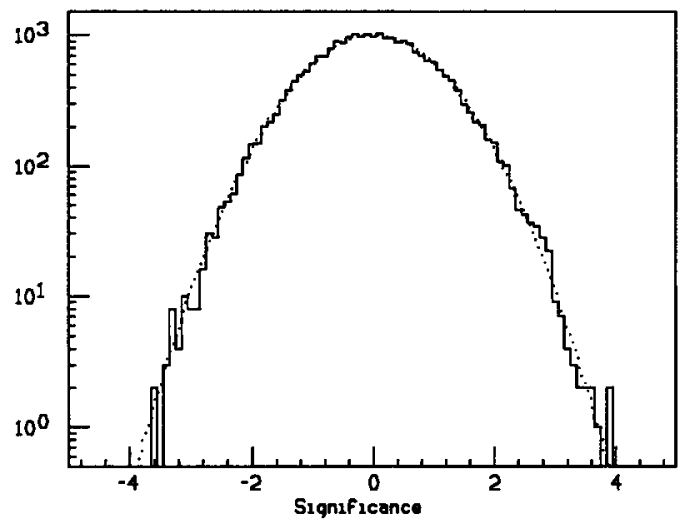

Figure 2. Distribution of bin significances for the muon poor sky (solid) overlaid with a best fit Gaussian (dashed).

short time, and the array yield peaks at the zenith, so this daily search effectively probes sources on a four hour time scale. Because of the large number of days to be searched, we a priori raise our detection threshold to require that at least one of the bins in a selected region contain at least a $5 \sigma$ excess.

We find five days on which a potential source appears and expect six from the random sky. None of these has a maximum significance larger than $5.8 \sigma$ and only one has a larger muon poor than all data significance. A $5.6 \sigma$ muon poor excess occurred on 29 April 1990 at $113^{\circ}$ right ascension and $61.6^{\circ}$ declination. This location has been examined for shorter term emission and the excess is found to be uniformly spread over the day. We consider these excesses to be completely consistent with normal background fluctuations.

We observe no evidence for point sources of gamma rays. This allows us to set stringent limits on the possible flux of gamma rays from such a source. Limits are calculated at the $90 \%$ confidence level by the following method. The exposure of the array to a search bin is determined by comparing the observed number of cosmic ray showers to the flux of cosmic rays into bin. The gamma ray exposure is obtained by correcting this measured cosmic ray exposure for the effects of mixed cosmic ray primary composition. A limit is placed on the possible number of gamma ray events from a source, based on our observation that the source search techniques utilized here are $90 \%$ efficient for finding $4.2 \sigma$ sources. The maximum possible gamma ray flux is then calculated under the assumption that the spectral index is the same (-1.6 integral) as the cosmic ray background. These limits are quoted at the median gamma ray energy which would be observed from each source.

Limits for continuous and daily gamma ray emission, as well as the energies to which they are appropriate, are shown in Figure 3. These limits apply to muon poor gamma ray emission. Limits on point sources of any particle which causes air showers, obtained without requiring muon poor showers, are a factor of 2.2 higher in each case.

\section{CONCLUSIONS}

We have searched a large fraction of the Northern sky for evidence of point sources of $100 \mathrm{TeV}$ gamma rays. Limits have been set on the possible flux from such a source at about $5.0 \times 10^{-14} \mathrm{~cm}^{-2} \mathrm{~s}^{-1}$. We have also searched for evidence of transient gamma ray emission. We set limits on the possible short term flux from point sources at about $1.0 \times 10^{-12} \mathrm{~cm}^{-2}$ $\mathrm{s}^{-1}$.

These flux limits are more than an order of magnitude below previously reported point source detections ${ }^{7,8}$. These early reports of source detections suggested that fluxes at these energies might be much higher than might be expected by extrapolating from lower energies. These limits rule out an anomalously bright $100 \mathrm{TeV}$ sky, and are now reaching the sensitivity required to see the kinds of fluxes predicted by extrapolation from lower energy. If we extrapolate the $10 \mathrm{TeV}$ flux of the Crab 


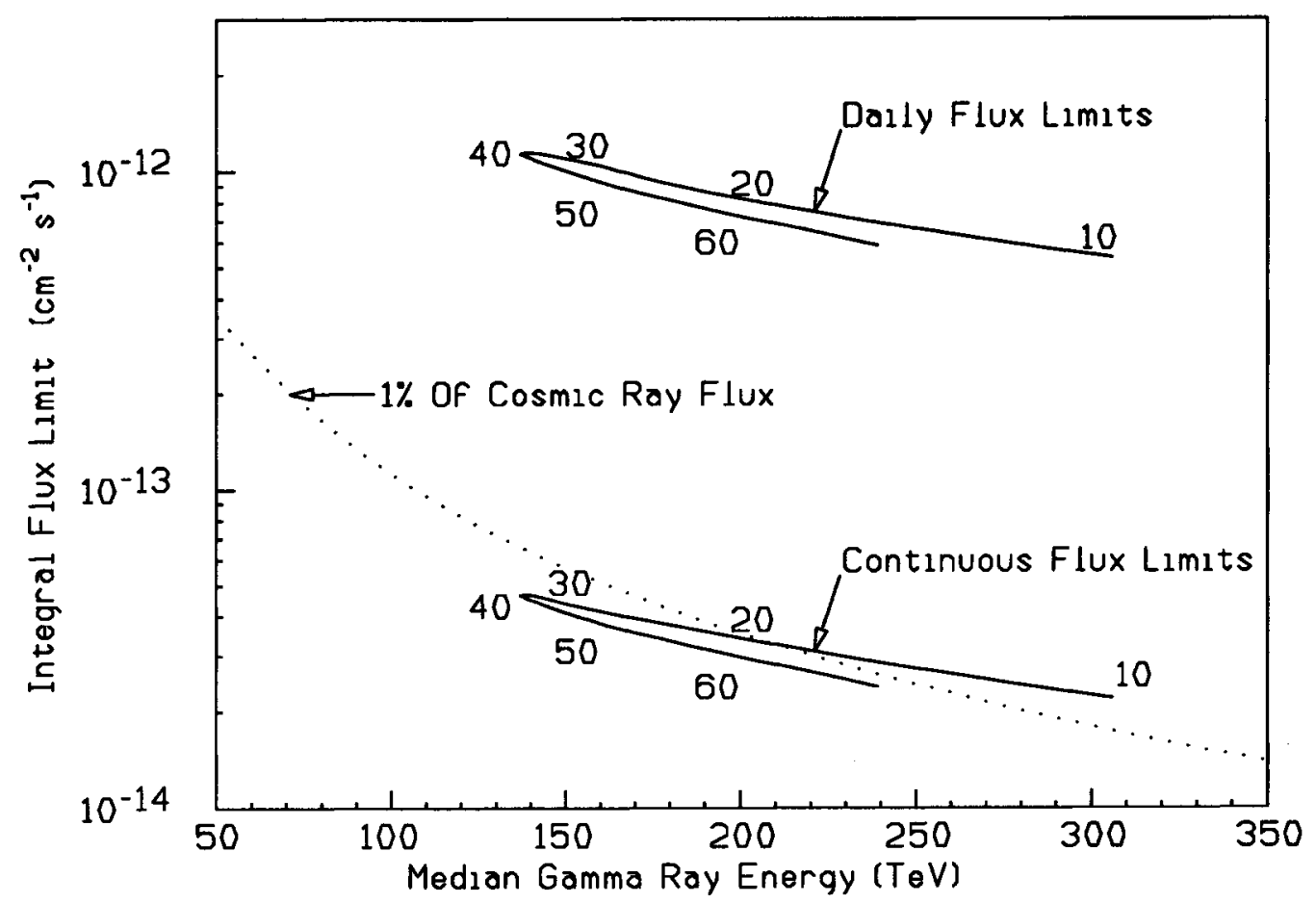

Figure 3. 90\% confidence limits on daily (top) and continuous (bottom) emission of gamma rays from point sources. Each curve describes the limit and median energy to which it is appropriate as a function of declination. Also shown is a curve representing $1 \%$ of the cosmic ray flux into the search bin.

Nebula to $100 \mathrm{TeV}$, for example, the expected flux is about $3.0 \times 10^{-14} \mathrm{~cm}^{-2} \mathrm{~s}^{-1}$. This flux level can certainly be reached in the near future.

The full CASA-MIA experiment is now operational. By the end of 1992 we will have accumulated about 900 million events. With the improved muon rejection of the full array the flux sensitivity will improve by a factor of two. Daily sensitivity has also improved by a factor of two. In a five year period of running the flux sensitivity will improve by more than a factor of four beyond that reported here.

\section{REFERENCES}

1. T.C. Weekes et al., Ap. J. 342, pp. 379395.
2. P. Baillon et al., Proc. of the XXVI Int. Conf. on High Energy Physics, 1992, in press.

3. A.M. Hillas, Proc. 19th Int. Cosmic Ray Conf., 1985, 3, 445.

4. R. Ong., Nuclear Physics B (Proc. Suppl.), 14A, 273 (1990).

5. D. Sinclair, Nucl. Instrum. Methods, A278, 583 (1989).

6. Ti-Pei Li and Yu-Qian Ma, Ap. J., 272, 317.

7. B.L. Dingus et al., Phys. Rev. Lett., 61, 1906 (1988).

8. M. Samorski and W. Stamm, Ap. J., 268 L17 (1983). 